\title{
GENERALIZATIONS OF THE WAVE EQUATION
}

\author{
J. MARSHALL ASH, JONATHAN COHEN, C. FREILING, AND DAN RINNE
}

ABSTRACT. The main result of this paper is a generalization of the property that, for smooth $u, u_{x y}=0$ implies

$$
u(x, y)=a(x)+b(y) \text {. }
$$

Any function having generalized unsymmetric mixed partial derivative identically zero is of the form $(*)$. There is a function with generalized symmetric mixed partial derivative identically zero not of the form $(*)$, but $(*)$ does follow here with the additional assumption of continuity.

These results connect to the theory of uniqueness for multiple trigonometric series. For example, a double trigonometric series is the $L^{2}$ generalized symmetric mixed partial derivative of its formal $(x, y)$-integral.

In this paper we introduce some generalized versions of the operator $\partial^{2} / \partial x \partial y$ and use them to extend the fact that for smooth real-valued functions on $\mathbf{R}^{2}$, $\partial^{2} f / \partial x \partial y=0$ implies that $f(x, y)=a(x)+b(y)$. In $\S 1$ we outline our results and in $\S 5$ we explain the connection of this material to the theory of uniqueness for multiple trigonometric series.

\section{INTRODUCTION}

The only global $C^{\infty}$ solutions to the wave equation $\partial^{2} f / \partial x \partial y=0$ are those of the form

$$
f(x, y)=a(x)+b(y) .
$$

(Write

$$
f(x, y)=\int_{0}^{y} \int_{0}^{x} \frac{\partial^{2} f}{\partial x \partial y}(s, t) d s d t+f(x, 0)+f(0, y)-f(0,0)
$$

to see this.) We want to replace the operator $\partial^{2} / \partial x \partial y$ with generalized operators $T$ which have the property that $T f=\partial^{2} f / \partial x \partial y$ for every $f$ in $C^{\infty}$. Two such operators are

$$
D f(x, y):=\lim _{h, k \rightarrow 0}\left\{\frac{f(x+h, y+k)-f(x+h, y)-f(x, y+k)+f(x, y)}{h k}\right\}
$$

Received by the editors April 9, 1990 and, in revised form, January 17, 1991. Presented January 18, 1990, American Mathematical Society Annual Winter Meeting at Louisville, in a 10 minute talk given by J. Marshall Ash entitled Generalized mixed partial derivatives.

1991 Mathematics Subject Classification. Primary 26B05; Secondary 42B99.

The first and second authors were supported in part by grants from the Faculty Research and Development Fund of the College of Liberal Arts and Sciences, DePaul University.

The third and fourth authors were supported in part by a Faculty Professional Development Grant, California State University, San Bernardino. 
and

(3)

$D_{s} f(x, y):=\lim _{h, k \rightarrow 0}\left\{\frac{f(x+h, y+k)-f(x+h, y-k)-f(x-h, y+k)+f(x-h, y-k)}{4 h k}\right\}$.

In these definitions $\lim _{h, k \rightarrow 0}$ means that the point $(h, k)$ tends to $(0,0)$ in such a way that $h$ and $k$ are never 0 , although the ratios $h / k$ and $k / h$ are uncontrolled.

If, motivated by the notion of restricted rectangular convergence, we control the ratios, then we arrive at two more generalized wave operators. Let $\Delta f(x, y ; h, k)$ and $\Delta_{s} f(x, y ; h, k)$ be the numerators of the curly bracketed terms in equations (2) and (3) respectively. If there is a number $s$ so that for every $M$, no matter how large,

$$
\lim _{\max \{|h|,|k|\} \rightarrow 0}\left(\sup _{1 / M \leq|h / k| \leq M}\left|\frac{\Delta f(x, y ; h, k)}{h k}-s\right|\right)=0,
$$

then we say that $f$ has a generalized $(x, y)$-derivative in the restricted sense and write $D^{r} f(x, y)=s$. If $\Delta$ is replaced by $\Delta_{s}$ in equation (4), we call $s$ the $(x, y)$-symmetric derivative in the restricted sense and write $D_{s}^{r} f(x, y)=$ $s$. We will see in $\S 2$ that all four operators $D, D_{s}, D^{r}$, and $D_{s}^{r}$ agree with $\partial^{2} / \partial x \partial y$ for sufficiently smooth functions. Another desirable property that such operators ought to enjoy is that for any decent, say Lebesgue measurable, function $f$, we ought to have

$$
D f(x, y)=0 \text { everywhere implies equation }(1),
$$

and

$$
D_{s} f(x, y)=0 \text { everywhere implies equation (1). }
$$

Now (5) does hold [5]. We will give a short proof ( $\$ 4$, Theorem 1$)$ of this below. However, it will be shown in $\S 3$ that a shock wave provides a counterexample to (6). Thus, to find some truth in (6) we must additionally assume that $f$ enjoys at least a little goodness $(\S 4$, Theorem 2$)$. Theorem 2 is by far the most difficult, and possibly the most interesting, result in this paper. Proposition 4 of $\S 3$ provides higher-dimensional examples of quite smooth functions on $\mathbf{R}^{d}$ that are not finite sums of functions of $d-1$ variables despite having the appropriate symmetric derivative everywhere 0 .

Pass now to multiple trigonometric series. For $\mathbf{x} \in \mathbf{R}^{d}$ and $\mathbf{n} \in \mathbf{Z}^{d}$, write $\mathbf{n x}:=\sum_{i=1}^{d} n_{i} x_{i}$ and $|\mathbf{n}|:=\sqrt{\sum n_{i}^{2}}$. If $\mathbf{x} \in \mathbf{R}^{d}$, we say that a trigonometric series $S(\mathbf{x})=\sum c_{\mathbf{n}} e^{i \mathbf{n} \mathbf{x}}$ converges unrestrictedly rectangularly at $\mathbf{x}$ to $s$ and write (R) $\sum c_{\mathbf{n}} e^{i \mathbf{n x}}=s$ if $\lim _{\min \left\{N_{j}\right\} \rightarrow \infty} \sum_{\left|n_{j}\right| \leq N_{j}, j=1, \ldots, d} c_{\mathbf{n}} e^{i \mathbf{n x}}=s$. For $d=2$, we define the formal $(x, y)$-integral of $S(\overline{\mathbf{x}})$ to be

$$
L(\mathbf{x}):=(\mathbf{R}) \sum^{\prime} \frac{c_{\mathbf{n}}}{(i m)(i n)} e^{i \mathbf{n} \mathbf{x}}+\left(\sum^{\prime} \frac{c_{m 0}}{i m} e^{i m x}\right) y+\left(\sum^{\prime} \frac{c_{0 n}}{i n} e^{i n y}\right) x+c_{00} x y .
$$

This is a formal $(x, y)$-integral of $S(\mathbf{x})$ in the sense that $\partial^{2} / \partial x \partial y$ applied to the nth term of $L$ produces $c_{\mathbf{n}} e^{i \mathbf{n x}}$. Say that $L$ has an $L^{2}$ symmetric mixed partial derivative at $(x, y)$ equal to $s$ if

$$
\lim _{(H, K) \rightarrow 0} \frac{1}{H K} \int_{0}^{H} \int_{0}^{K}\left|\frac{\Delta_{s} L(x, y ; h, k)}{4 h k}-s\right|^{2} d h d k=0 .
$$


When this is the case write $D_{s, 2} L(x, y)$ for $s$.

In $\S 5$ we will motivate and prove the following two theorems.

Theorem 3. If (R) $\sum c_{\mathbf{n}} e^{i \mathbf{n} \mathbf{x}}=s$ for a fixed $\mathbf{x}$ in $\mathbf{R}^{2}$, the rectangular partial sums are bounded, and the coefficients are bounded, then $D_{s, 2} L(\mathbf{x})=s$.

Theorem 4. If, for $x \in \mathbf{R}$, the Fourier series $\sum c_{n} e^{i n x}$ satisfies $c_{n}=o\left(\frac{1}{n}\right)$, then the associated function is of vanishing mean oscillation.

The last section makes explicit what remains to be done in order to reach a uniqueness theorem for multiple trigonometric series based on considerations of the generalized wave equation.

\section{Relations}

The relations at a fixed point between varying degrees of smoothness and the existence of the four generalized mixed partials are exhibited in Figure 1. Take the fixed point to be $\mathbf{0}=(0,0)$. Then $f_{x}$ means $\frac{\partial f}{\partial x}(\mathbf{0}), f_{x y}$ means $\frac{\partial^{2} f}{\partial y \partial x}(\mathbf{0})$, and the existence of the second Peano derivative means that there are constants $a, b, c$ so that

$$
f(h, k)=f(\mathbf{0})+f_{x}(\mathbf{0}) h+f_{y}(\mathbf{0}) k+a \frac{h^{2}}{2}+b h k+c \frac{k^{2}}{2}+o\left(h^{2}+k^{2}\right)
$$

as $(h, k)$ tends to $\mathbf{0}$.

Six of the eleven implications shown in Figure 1 are trivial. That 1 implies 4 follows from Peano's theorem (see [3, Theorem 4]). That 6 implies 8 and that 7 implies 9 follows from the identity

$\Delta_{s} f(\mathbf{0} ; h, k)=\Delta f(\mathbf{0} ; h, k)+\Delta f(\mathbf{0} ;-h, k)+\Delta f(\mathbf{0} ; h,-k)+\Delta f(\mathbf{0} ;-h,-k)$.

The remaining two implications, 4 implies 7 and 2 implies 6, are Propositions 1 and 2 below.

Of course several other implications between the nodes of Figure 1, such as 1 implies 6 , follow by concatenation of the implications shown. However, beyond these, no other implications hold. To show this we will give below seven simple

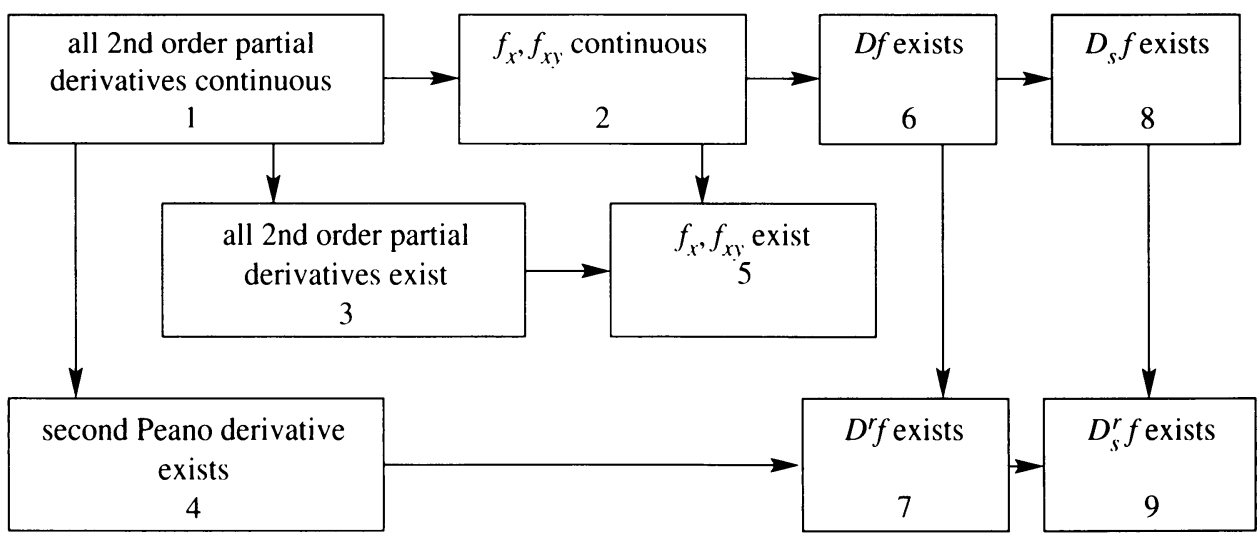

FIGURE 1 
example functions $A, B, C, \ldots, G$. Their relevant properties are summarized in the following table:

$\begin{array}{llllllllll} & 1 & 2 & 3 & 4 & 5 & 6 & 7 & 8 & 9 \\ 1 & & & & & & & & & \\ 2 & F & & F & F & & & & & \\ 3 & E & E & & E & & E & E & E & E \\ 4 & D & D & D & & D & D & & D & \\ 5 & F & G & F & F & & E & E & E & E \\ 6 & F & C & F & F & C & & & & \\ 7 & F & C & F & F & C & A & & A & \\ 8 & F & C & F & F & C & B & B & & \\ 9 & F & C & F & F & C & A & B & A & \end{array}$

Here for example, the $G$ in the $(5,2)$ position of the table means that the function $G$ has $G_{x}(\mathbf{0})$ and $G_{x y}(\mathbf{0})$ existing (property 5 of Figure 1) but $G_{x}$ and $G_{x y}$ are not continuous at $\mathbf{0}$ (property 2 of Figure 1 failing). A blank entry in the table means that the corresponding implication can be read off from Figure 1. For example, the blank in position $(4,9)$ means that the existence of the second Peano derivative at $\mathbf{0}$ (property 4 ) does imply the existence of $D_{s}^{r} f(\mathbf{0})$ (property 9). The example functions are

$$
\begin{aligned}
& A(x, y):=\text { the characteristic function of }\left\{\left(x, x^{2}\right): x>0\right\}, \\
& B(x, y):=|x| y, \\
& C(x, y):=|x|+|y|, \\
& D(x, y):=y^{3} \text { times the characteristic function of }\{(x, y): x>0\}, \\
& E(x, y):=x y \frac{x^{2}-y^{2}}{x^{2}+y^{2}}, \quad x^{2}+y^{2} \neq 0, \quad E(0):=0, \\
& F(x, y):=|y|, \\
& G(x, y):=\left(x^{2} \sin \frac{1}{x}\right) y, \quad x \neq 0, \quad G(0, y):=0 .
\end{aligned}
$$

To complete Figure 1 we need only confirm that property 4 implies property 7 and that property 2 implies property 6.

Proposition 1. Let $f: \mathbf{R}^{2} \rightarrow \mathbf{R}$ have two Peano derivatives at $\mathbf{p}$. Then $D^{r} f(\mathbf{p})$ exists.

Proof. We have

$$
f(\mathbf{p}+(h, k))=f(\mathbf{p})+f_{x}(\mathbf{p}) h+f_{y}(\mathbf{p}) k+\frac{a}{2} h^{2}+b h k+\frac{c}{2} k^{2}+o\left(h^{2}+k^{2}\right) .
$$

Direct substitution into $\Delta_{s}(\mathbf{p}, h, k)$ shows that

$$
\frac{\Delta_{s}(\mathbf{p}, h)}{4 h k}=\frac{4 b h k+o\left(h^{2}+k^{2}\right)}{4 h k} ;
$$

but

$$
\frac{o\left(h^{2}+k^{2}\right)}{h k}=o\left(\frac{h}{k}\right)+o\left(\frac{k}{h}\right)=o(1)
$$

if the ratio $\left|\frac{h}{k}\right|$ is bounded above and below.

Remark. Note that the example $D(x, y)$ given above shows that the $r$ in the conclusion of Proposition 1 is essential. 
Proposition 2. Let $f_{x}$ and $f_{x y}$ be continuous at $\mathbf{p}$. Then $D f(\mathbf{p})=f_{x y}(\mathbf{p})$.

Proof. Pick $h$ and $k$ so small that $f_{x}$ and $f_{x y}$ exist throughout the rectangle with corners $\mathbf{p}+( \pm h, \pm k)$. Now fix $\mathbf{p}$ and $k$ and set

$$
g(t):=f(\mathbf{p}+(t, k))-f(\mathbf{p}+(t,-k)) \text { for }-|h| \leq t \leq|h| .
$$

By the mean value theorem, $\Delta(\mathbf{p} ; h, k)=g(h)-g(-h)=g_{x}(\theta h) 2 h$, where $\theta \in(-1,1)$. Again

$$
g_{x}(\theta h)=f_{x}(\mathbf{p}+(\theta h, k))-f_{x}(\mathbf{p}+(\theta h,-k))=f_{x y}(\mathbf{p}+(\theta h, \varphi k)) 2 k,
$$

where $\varphi \in(-1,1)$. Combining these two results gives

$$
\Delta(\mathbf{p} ; h, k)=f_{x y}(\mathbf{p} ; \theta h, \varphi k) 4 h k .
$$

Divide by $4 h k$ and let $h$ and $k$ go independently to $(0,0)$.

Remark. This proof is well known.

\section{SHOCK WAVES}

A simple shock wave is the function

$$
S(x, y):=\left\{\begin{array}{ll}
1 & \text { if }|y|>|x| \\
0 & \text { if }|y|=|x| \\
-1 & \text { if }|y|<|x|
\end{array}\right\}=\operatorname{sgn}(|y|-|x|) .
$$

If $x \neq y, \Delta_{s}(x, y ; h, k)$ is 0 for all small $h$ and $k$ since all four terms $S(x \pm h, y \pm k)$ are equal; if $x=y$, considering separately the cases $|h|>|k|$, $|h|=|k|$, and $|h|<|k|$, shows that $\Delta_{s}(x, y, h, k)$ is 0 for all choices of $(h, k)$. Hence, $D_{s} S(x, y)$ is identically 0 . A function of the form $a(x)+b(y)$ is the same function of $x$, up to a vertical translation, for each fixed choice of $y$. Clearly $S(x, y)$ does not have this property.

Proposition 3. There is a bounded, almost everywhere continuous, $2 \pi$-periodic in each variable function $S_{1}(x, y)$ satisfying (i) $D_{s} S_{1}(x, y)=0$ for all $(x, y)$ and (ii) $S_{1}$ is not of the form $a(x)+b(y)$.

Proof. All we need is a periodized version of the function $S(x)$. For $(x, y) \in$ $T^{2}:=(-\pi, \pi] \times(-\pi, \pi]$ let $S_{1}(x, y):=\operatorname{sgn}(|y|-|x|)$ and complete the definition of $S_{1}$ by extending it to be $2 \pi$-periodic in each variable. Direct calculation of Fourier coefficients shows that

$$
S_{1}(x, y)=\frac{2}{\pi}(|y|-|x|)+L(x, y),
$$

where

$$
\begin{aligned}
L(x, y) & :=\frac{16}{\pi^{2}} \sum_{n \neq m(\bmod 2)}^{\prime} \frac{1}{m^{2}-n^{2}} \cos m x \cos n y \\
& =-\frac{8}{\pi^{2}} \sum_{m=1}^{\infty} \sum_{\substack{n=1 \\
n \neq m(\bmod 2)}}^{\infty} \frac{1}{m}\left(\frac{1}{m+n}+\frac{1}{m-n}\right) \cos m x \cos n y .
\end{aligned}
$$

As with $S(x, y)$ above, a glimpse at the graph of $S_{1}$ shows that it is not of the form $a(x)+b(y)$ (see Figure 2). That $D_{s} S_{1} \equiv 0$ is shown using the same case-by-case analysis that was used for $S$ above. 


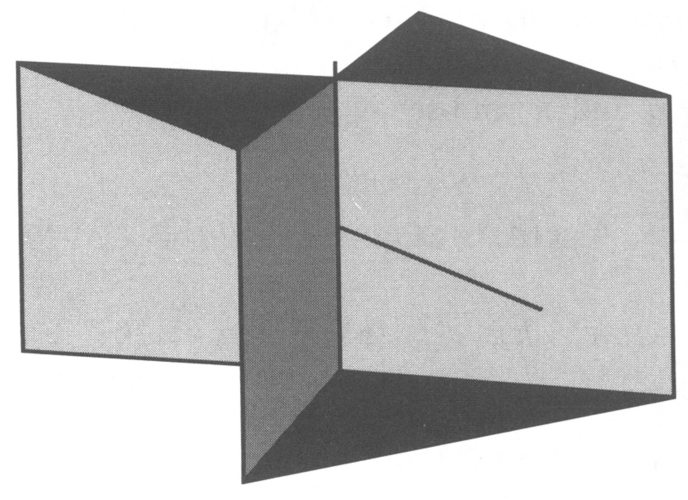

$S(x, y)$

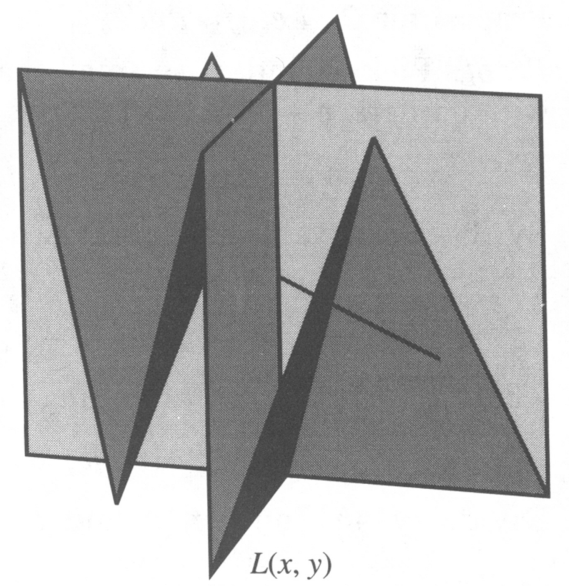

FIGURE 2

Remark. The periodic function $L(x, y)$ also supplies an example satisfying the condition of Proposition 3. This is immediate since $L$ and $S_{1}$ differ by a function of the form $a(x)+b(y)$.

Remark. Let $F(x, y):=\int_{0}^{x} \int_{0}^{y} L(s, t) d s d t$. The periodic function $F$ provides a counterexample to a 1974 conjecture of Ash and Welland [4, p. 427]. More precisely,

$$
\lim _{h, k \rightarrow 0}\left\{\begin{array}{l}
F(x+h, y+k)-2 F(x+h, y)+F(x+h, y-k) \\
-2 F(x, y+k)+4 F(x, y)-2 F(x, y-k) \\
+F(x-h, y+k)-2 F(x-h, y)+F(x-h, y-k)
\end{array}\right\} h^{-2} k^{-2}=0,
$$

everywhere even though $F$ is not of the form $a(x) y+b(y) x+c(y)+d(x)$.

Passing to higher dimensions, let $\varepsilon_{i}=1$ or $-1, i=1,2, \ldots, d$, and let $E=E(d)$ be the set of all $2^{d} \boldsymbol{\varepsilon}$ 's. Define a coordinatewise product by $\boldsymbol{\varepsilon} \bullet \mathbf{h}:=\left(\varepsilon_{1} h_{1}, \varepsilon_{2} h_{2}, \ldots, \varepsilon_{d} h_{d}\right)$. Define $\pi(\mathbf{h}):=h_{1} h_{2} \cdots h_{d}$ and

$$
\mathscr{D}_{d} f(\mathbf{x}):=\lim _{\substack{\mathbf{h} \rightarrow 0 \\ \pi(\mathbf{h}) \neq 0}} \frac{\sum_{\boldsymbol{\varepsilon} \in E} \pi(\boldsymbol{\varepsilon}) f(\mathbf{x}+\boldsymbol{\varepsilon} \bullet \mathbf{h})}{\pi(2 \mathbf{h})} .
$$

Note that if $d=2$, then $\mathscr{D}_{2} f(\mathbf{x})=D_{s} f(\mathbf{x})$. Observe that for sufficiently smooth $f: \mathbf{R}^{d} \rightarrow \mathbf{R}, \partial^{d} f / \partial x_{1} \partial x_{2} \cdots \partial x_{d}=0$ implies $f(\mathbf{x})=f_{1}\left(x_{2}, \ldots, x_{d}\right)+$ $f_{2}\left(x_{1}, x_{3}, \ldots, x_{d}\right)+\cdots+f_{d}\left(x_{1}, \ldots, x_{d-1}\right)$. It is easy to see that for sufficiently smooth $f, \mathscr{D}_{d} f(\mathbf{x})=\left(\partial^{d} f / \partial x_{1} \partial x_{2} \cdots \partial x_{d}\right)(\mathbf{x})$. However, we have the following set of examples.

Proposition 4. For each dimension $d \geq 3$, there is a $C^{d-3}\left(\mathbf{R}^{d}\right)$ function $F_{d}$ satisfying (i) $\mathscr{D}_{d} F_{d}(\mathbf{x})=0$ for all $\mathbf{x}$ and (ii) $F_{d}$ is not a finite sum of functions of $d-1$ variables.

Proof. Let

$$
F_{d}(\mathbf{x}):=\left(\sum_{i=1}^{d} x_{i}\right)^{d-2} \operatorname{sgn}\left(\sum_{i=1}^{d} x_{i}\right)
$$


It is easy to check that $F_{d}$ is $C^{d-3}$ and that $F_{d}$ has property (ii). We now show that (i) holds. If $\sum_{i=1}^{d} x_{i} \neq 0$, then $F_{d}$ is infinitely differentiable at $\mathbf{x}$, so it is immediate that

$$
\mathscr{D}_{d} F_{d}(\mathbf{x})=\frac{\partial^{d}}{\partial x_{1} \partial x_{2} \cdots \partial x_{d}} F_{d}(\mathbf{x})=0 .
$$

If $\sum x_{i}=0$, then

$$
\begin{aligned}
\sum_{\boldsymbol{\varepsilon} \in E} \pi(\boldsymbol{\varepsilon}) F_{d}(0+\boldsymbol{\varepsilon} \bullet \mathbf{h}) & =\sum_{\boldsymbol{\varepsilon} \in E} \pi(\boldsymbol{\varepsilon})\left(\sum_{i=1}^{d} \varepsilon_{i} h_{i}\right)^{d-2} \operatorname{sgn}\left(\sum_{i=1}^{d} \varepsilon_{i} h_{i}\right) \\
= & \frac{1}{2}\left[\sum_{\boldsymbol{\varepsilon} \in E} \pi(\boldsymbol{\varepsilon})\left(\sum_{i=1}^{d} \varepsilon_{i} h_{i}\right)^{d-2} \operatorname{sgn}\left(\sum_{i=1}^{d} \varepsilon_{i} h_{i}\right)\right. \\
& \left.\quad+\sum_{-\boldsymbol{\varepsilon} \in E} \pi(-\boldsymbol{\varepsilon})\left(\sum_{i=1}^{d}-\varepsilon_{i} h_{i}\right)^{d-2} \operatorname{sgn}\left(\sum_{i=1}^{d}-\varepsilon_{i} h_{i}\right)\right] \\
= & 0, \quad
\end{aligned}
$$

since $\pi(-\boldsymbol{\varepsilon})=(-1)^{d} \pi(\boldsymbol{\varepsilon}),\left(\sum_{i=1}^{d}-\varepsilon_{i} h_{i}\right)^{d-2}=(-1)^{d-2}\left(\sum_{i=1}^{d} \varepsilon_{i} h_{i}\right)^{d-2}$, and $\operatorname{sgn}\left(\sum_{i=1}^{d}-\varepsilon_{i} h_{i}\right)=(-1) \operatorname{sgn}\left(\sum_{i=1}^{d} \varepsilon_{i} h_{i}\right)$. Consequently, $\mathscr{D}_{d} F_{d}(\mathbf{x})=0$ here also.

Remark. Note that it is also possible to let $d=2$ in the above argument, thereby producing a function similar to $S(x, y)$.

\section{Positive differentiation Results}

Theorem 1. If $D f(x, y)=0$ for all $(x, y)$, then there are one-variable functions $a(x)$ and $b(y)$ so that $f(x, y)=a(x)+b(y)$.

Remarks. Note that nothing, not even measurability, is presumed about $f$ 's goodness. The proof given below is not substantially different from Bogel's earlier one, but we believe that our shorter presentation will make the ideas more accessible, at least to readers more comfortable with English than German [5].

Proof of Theorem 1. Given any rectangle $R$, with "southwest" corner $(x, y)$ and "northeast" corner $(x+h, y+k)$, write $\delta(R)=\Delta f(x, y ; h, k) / 4 h k$. Let $R$ be quadrasected by a vertical and a horizontal line passing through $\mathbf{p}=$ $(\theta x+(1-\theta)(x+h), \varphi y+(1-\varphi)(y+h)), 0<\theta<1,0<\varphi<1$. This creates four subrectangles $A, B, C, D$. This proof will be accomplished by exploiting the simple algebraic equality

$$
\delta(R)=\frac{|A|}{|R|} \delta(A)+\frac{|B|}{|R|} \delta(B)+\frac{|C|}{|R|} \delta(C)+\frac{|D|}{|R|} \delta(D) .
$$

This equation asserts that $\delta(R)$ is a convex combination of $\delta(A), \delta(B), \delta(C)$, and $\delta(D)$.

Replacing $f$ by $f(x, y)-\{f(x, 0)-f(0,0)\}-\{f(0, y)\}$ allows us to assume that $f=0$ on both coordinate axes. Now it suffices to prove that $f=0$ 
everywhere. Suppose that there is a fixed point $(x, y)$, with $f(x, y) \neq 0$. Without loss of generality, $f(x, y)>0$.

Let $R_{0}$ be the rectangle with corners at $(0,0)$ and $(x, y)$. Symmetrically quadresect $R_{0}$. Upon setting $a:=f(x, y) /\left|R_{0}\right|$, from (7) we have

$$
a=\delta\left(R_{0}\right)=\frac{1}{4} \delta(A)+\frac{1}{4} \delta(B)+\frac{1}{4} \delta(C)+\frac{1}{4} \delta(D),
$$

say, where $R_{0}=A \cup B \cup C \cup D$ and $|A|=|B|=|C|=|D|=\frac{1}{4}\left|R_{0}\right|$. One of the four $\delta$ 's on the right side must be $\geq a$. Call it $\delta_{1}$ and call the corresponding rectangle $R_{1}$. Similarly write $\delta_{1}$ as an average of four $\delta$ 's and select $\delta_{2}$ and $R_{2}$ in the same way. In particular, $\delta_{2}\left(R_{2}\right) \geq a$ and $\left|R_{2}\right|=\left(\frac{1}{4}\right)^{2}\left|R_{0}\right|$. Iterate this process to produce a shrinking nested sequence of similar (in the sense of Euclidean geometry) rectangles that rapidly shrink to a point $\mathbf{p}$.

Now quadrasect $R_{j}$ with horizontal and vertical lines through $\mathbf{p}$. Again apply (7), select a $\delta$ satisfying $\delta \geq a$, call it $\tilde{\delta}_{j}$, and call the corresponding . rectangle $\widetilde{R}_{j}$. (Note that if $\mathbf{p}$ lies on an edge of $R_{j}$, then $\delta_{j}$ is a convex combination of only two $\delta$ 's, the larger of which becomes $\tilde{\delta}_{j}$; and if $\mathbf{p}$ is in a corner of $R_{j}$, then $\tilde{\delta}_{j}$ is $\delta_{j}$.) Then $\widetilde{R}_{j} \subset R_{j}$ so the $\widetilde{R}_{j}$ 's also shrink to $\mathbf{p}$. Since $\mathbf{p}$ is a corner of $\widetilde{R}_{j}, \tilde{\delta}_{j}$ may be written as $\Delta f\left(\mathbf{p} ; h_{j}, k_{j}\right) / 4 h_{j} k_{j}$. Then

$$
\lim _{j \rightarrow \infty} \inf \tilde{\delta}_{j} \geq a
$$

contrary to $D f(\mathbf{p})=0$.

Remark. The ratio of the side lengths is the same for every $R_{j}$. However, this ratio may be unbounded for the $\widetilde{R}_{j}$ 's so $D^{r}(\mathbf{p})=0$ is not contradicted by (8). Thus the proof of Theorem 3 gives no insight into the question of whether $D^{r} f(x, y)=0$ for all $(x, y)$ forces $f(x, y)=a(x)+b(y)$. Actually, using very delicate real variable techniques, Buczolich has proved that if

$$
\lim _{\max \{|h|,|k|\} \rightarrow 0}\left(\sup _{1 / 1000 \leq|h / k| \leq 1000}\left|\frac{\Delta f(x, y ; h, k)}{h k}-s\right|\right)=0
$$

(obviously a far weaker condition than $D^{r} f(\mathbf{p})=0$ ) for every $\mathbf{p}$, then $f(x, y)$ $=a(x)+b(y)$. (To see this, first reduce to a statement like Lemma 1 below in just the same way as Theorem 2 below is reduced to Lemma 1 . The resultant lemma then follows almost immediately from Theorem 1.1 of [6]. This theorem asserts that if $\delta$ is a positive-valued function on a closed rectangle, then there is a partition of that rectangle into a finite number of rectangles $\left\{A_{i}\right\}$ such that the eccentricity of each $A_{i}$ is no worse than $\frac{1}{1000}$ and such that each $A_{i}$ has a corner $x_{i}$ with the disk of radius $\delta\left(x_{i}\right)$ centered at $x_{i}$ containing $A_{i}$.)

Theorem 2. If $D_{s} f(x, y)=0$ for all $(x, y)$ and if $f$ is continuous, then there are one-variable functions $a(x)$ and $b(y)$ so that $f(x, y)=a(x)+b(y)$.

Remarks. In one dimension, define $\lim _{h \rightarrow 0}(f(x+h)-f(x-h)) / 2 h$ to be the first symmetric derivative of $f$ at $x$. Khintchine has pointed out that a continuous function on $\mathbf{R}^{1}$ with an everywhere 0 first symmetric derivative is constant [ 10 , pp. 215-216]. Theorem 2 seems to be a natural generalization of Khintchine's result. 
In one dimension, the characteristic function of a single point has everywhere 0 first symmetric derivative so that some condition similar to continuity is needed. Similarly here in two dimensions, the examples $S(x, y)$ and $F_{2}(x, y)$ given in $\S 3$ show that some condition similar to continuity is needed.

We note that the roof given below cannot readily be extended to dimension 3 or more, since the example $F_{3}$ of Proposition 4 is continuous.

Proof of Theorem 2. It is sufficient to prove that $\Delta_{s} f(x, y ; h, k)=0$ for every $x, y, h$, and $k$. Replacing $f(x, y)$ by $f(x, y)-\{f(x, 0)+f(0, y)-f(0,0)\}$ shows that, without loss of generality, we may adjoin the hypothesis that $f$ vanishes on the coordinate axes and then try to show that $f$ vanishes directly. But then $f(x, y)=\Delta_{s} f\left(\frac{x}{2}, \frac{y}{2} ; \frac{x}{2}, \frac{y}{2}\right)=0$. Since $\Delta_{s} f(x, y ; \cdot, k)$ and $\Delta_{s} f(x, y ; h, \cdot)$ are both odd functions of a single real variable, it is sufficient to prove

$$
\begin{aligned}
\Delta_{s} f(x, y ; h, k)=0 & \text { for every }(x, y) \text { and every positive } h \\
& \text { and positive } k .
\end{aligned}
$$

Now fix $\varepsilon>0$, and note that $D_{s} x y=\left(\partial^{2} / \partial x \partial y\right) x y=1$ so that $D_{s}(f+\varepsilon x y)=\varepsilon>0$. By Lemma 1 below, $\Delta_{s}(f+\varepsilon x y)(x, y ; h, k) \geq 0$ for every $(x, y)$ and every positive $h$ and positive $k$. Fix $(x, y), h$, and $k$ and let $\varepsilon \rightarrow 0$ to get equation (9) with $=$ replaced by $\geq$. Then repeat this argument with $f$ replaced by $-f$ to get equation (9) with $=$ replaced by $\leq$.

Lemma 1. Let $f: \mathbf{R}^{2} \rightarrow \mathbf{R}$ be continuous and assume that there is a constant $c$ such that for every $(x, y)$ in $\mathbf{R}^{2}$

$$
D_{s} f(x, y) \geq c>0 .
$$

Then $\Delta_{s} f(x, y ; h, k) \geq 0$ for every $(x, y)$ and every positive $h$ and positive $k$.

Proof. Fix a function $f$ satisfying the hypothesis of the lemma. By a box we will mean a closed nondegenerate rectangle with sides parallel to the axes. For a box $B:=[a, b] \times[c, d]$, let $\Delta_{s} B:=f(b, d)+f(a, c)-f(a, d)-f(b, c)$. A simple identity that will be used repeatedly in this proof is

$$
\begin{aligned}
& \text { If the box } B \text { is a finite union of nonoverlapping boxes } B_{i}, \\
& \text { then } \Delta_{s} B=\sum_{i} \Delta_{s} B_{i} \text {. }
\end{aligned}
$$

For any set $A$ let $A^{\circ}$ be the interior of $A$, let $\bar{A}$ be the closure of $A$, and let $\partial A$ be the boundary of $A$. It is well known that $\partial A$ is closed and if $A$ is closed, then $\partial A$ is also nowhere dense. We will first show that there is a set $S$ satisfying

$$
S \text { is an open set, }
$$

$$
\bar{S}=\mathbf{R}^{2},
$$

and

$$
\Delta_{s} B \geq 0 \text { for every box } B \subset S \text {. }
$$


Since $f$ is continuous, for each choice of $h$ and $k$,

$$
B_{h k}:=\left\{(x, y): \Delta_{s} f(x, y ; h, k) \geq 0\right\}
$$

is closed so that, for each positive integer $n$, the set

$$
\begin{aligned}
A_{n} & :=\left\{(x, y): \Delta_{s} f(x, y ; h, k) \geq 0 \text { for all }(h, k) \in\left(0, \frac{1}{n}\right]^{2}\right\} \\
& =\bigcap\left\{B_{h k}:(h, k) \in\left(0, \frac{1}{n}\right]^{2}\right\}
\end{aligned}
$$

is also closed. Clearly $A_{n} \nearrow$ and from assumption (10) we have $\bigcup A_{n}=\mathbf{R}^{2}$.

Let $S:=\bigcup\left(A_{n}\right)^{\circ}$. Clearly $S$ satisfies (12). Now $\mathbf{R}^{2}=\bigcup A_{n}=$ $\bigcup\left(A_{n}^{\circ} \cup \partial A_{n}\right)=S \cup\left(\bigcup \partial A_{n}\right)$ and since each $\partial A_{n}$ is closed and nowhere dense the Baire Category Theorem asserts that the complement of $\bigcup \partial A_{n}$ is dense in $\mathbf{R}^{2}$. Since $S$ contains that complement, (13) follows. To see that $S$ satisfies (14) let $B$ be a box contained in $S$. Since $B$ is compact it is contained in the union of finitely many $A_{n}^{\circ}$ 's. But the $A_{n}$ 's are nested, so that $B$ is contained in a single $A_{n}$. If the dimensions of $B$ are both $\leq \frac{1}{n}$, then $\Delta_{s} B \geq 0$ by definition of $A_{n}$; while if $B$ is too large, simply write it as a finite union of small (dimensions $\leq \frac{1}{n}$ ) nonoverlapping boxes and combine the definition of $A_{n}$ with property (11) to conclude that $S$ satisfies (14).

Now let $T$ be the union of all sets $S$ satisfying conditions (12), (13), and (14). Obviously $T$ itself satisfies (12) and (13). We now show that $T$ satisfies (14). Let $B$ be a box contained in $T$. Each point in $B$ is the center of a box $D$ contained in an $S$ satisfying (14). But $B$ is compact, so finitely many such $D$ cover $B$. By extending the edges of these $D$ until they reach the edge of $B$ we may produce a decomposition of $B$ into a finite collection of nonoverlapping boxes $\left\{B_{i}\right\}$ such that each $B_{i}$ is contained in a $D$ and consequently in an $S$ satisfying (14). Apply (11) again to see that $T$ satisfies (14).

If $T=\mathbf{R}^{2}$, Lemma 1 is proved. If not, let $C$ be the closed nowhere dense complement of $T$. Then $C=\bigcup\left(A_{n} \cap C\right)$, so by the Baire Category Theorem some $A_{n}$ contains a portion of $C$. Let $B$ be a small (dimensions $\leq \frac{1}{n}$ ) box inside this portion such that $B^{\circ} \cap C$ is not empty. We now show that $T \cup B^{\circ}$ satisfies (12), (13), and (14), thereby contradicting the definition of $T$ and establishing Lemma 1.

That properties (12) and (13) hold is clear, so let $D$ be a box, $D \subset T \cup B^{\circ}$. Then $D$ is decomposable into $D \cap B$ and a union of at most four other boxes, each of which is entirely contained in $T$. Property (14) and hence the desired contradiction will follow from identity (12) provided we can show that $\Delta_{s} D \cap$ $B \geq 0$. Rename $D \cap B$ as $B$ and complete the proof by applying the following lemma.

Lemma 2. Let $B$ be a box which meets the closed nowhere dense set $C$ such that, for every box $B^{\prime} \subset B$,

$$
B^{\prime} \text { disjoint from } C \text { implies } \Delta_{s} B^{\prime} \geq 0
$$

and

$$
\text { center of } B^{\prime} \text { in } C \text { implies } \Delta_{s} B^{\prime} \geq 0 \text {. }
$$

Then $\Delta_{s} B \geq 0$. 
Proof. For ease of calculation we assume that $B$ is centered at $(0,0)$ and has first quadrant vertex $(a, b)$. Let $Y$ be the $y$-axis. We distinguish three cases.

Case 1: $Y \cap B \cap C=\varnothing$. For $0<c \leq a$, let $N(c)$ be the box with vertices $(0,-b),(c,-b),(c, b)$, and $(0, b)$. Since $Y \cap B$ and $C$ are disjoint closed sets, they are separated by some positive distance. Thus, for some $c^{\prime}>0$, $N\left(c^{\prime}\right) \cap C=\varnothing$, and, for any $B^{\prime} \subset N\left(c^{\prime}\right)$, we have $\Delta_{s} B^{\prime} \geq 0$. Let $S=$ $\left\{c \mid 0<c \leq a\right.$ and $\Delta_{s} B^{\prime} \geq 0$ for any $B^{\prime} \subset N(c)$ with left edge in $\left.Y\right\}$ and let $\tau=\sup S$. By the selection of $c^{\prime}$ above, $S \neq \varnothing$, and the continuity of $f$ implies that $\tau \in S$. Suppose that $\tau<a$ and let $t:=\min \{\tau,(a-\tau) / 2\}$. We show that this gives $\tau+t \in S$, contradicting the choice of $\tau$. It suffices to show $\Delta_{S} N(\tau+t) \geq 0$, since the same argument will apply to any box contained in $N(\tau+t)$ with left edge contained in $Y$.

Let $V_{1}$ be the box with opposite corners $(\tau,-b)$ and $(\tau+t / 2, b)$. If $V_{1}^{\circ} \cap$ $C \neq \varnothing$, pick $\left(x_{1}, y_{1}\right) \in V_{1} \cap C$ closest to the horizontal bisector of $V_{1}$. Let $B_{1}$ be the largest box centered at $\left(x_{1}, y_{1}\right)$ that is contained in $N(\tau+t)$ and let $B_{1}^{\prime}$ be the box that has the same right edge as $B_{1}$, and has left edge in $Y$. By (11), $\Delta_{s} B_{1}^{\prime} \geq 0$ since $\Delta_{s} B_{1} \geq 0$ and the left edge of $B_{1}$ is in $N(\tau)$. Note that the right edge of $B_{1}$ is contained in the right edge of $N(\tau+t)$. Now suppose $V_{k},\left(x_{k}, y_{k}\right), B_{k}$, and $B_{k}^{\prime}, k=1, \ldots, n-1$, have been selected. We define box $V_{n}=\overline{V_{n-1} \backslash B_{n-1}}$. If $V_{n}^{\circ} \cap C \neq \varnothing$, pick $\left(x_{n}, y_{n}\right) \in V_{n} \cap C$ closest to the horizontal bisector of $V_{n}$. Let $B_{n}$ be the largest box centered at $\left(x_{n}, y_{n}\right)$ that is contained in the closure of $N(\tau+t) \backslash \bigcup_{k=1}^{n-1} B_{k}$ and let $B_{n}^{\prime}$ be the box that has the same right edge as $B_{n}$, and has left edge in $Y$. If some $V_{n}^{\circ} \cap C=\varnothing$, we stop and obtain a finite sequence of boxes $\left\{B_{n}^{\prime}\right\}$. Otherwise, the sequence is infinite. If $\overline{\cup B_{n}^{\prime}}=N(\tau+t)$, then, by the continuity of $f$, we are done. Otherwise, $N(\tau+t)$ is the union of at most four nonoverlapping boxes $O_{1}, U_{1}, L_{1}$, and $R_{1}$, of the following form. ( $O$ is for "over," $U$ for "under," $L$ for "left," and $R$ for "right.") Observe that $\left\{B_{n}^{\prime}\right\}$ can be split into two disjoint collections of boxes. The union of the boxes in one collection forms the box $O_{1}$ and contains the top edge of $N(\tau+t)$. The other union similarly forms the box $U_{1}$ and contains the bottom edge of $N(\tau+t)$. Let $L_{1}$ (respectively $R_{1}$ ) be the portion of $\overline{N(\tau+t) \backslash\left(O_{1} \cup U_{1}\right)}$ to the left (respectively right) of the vertical line $x=\tau+t / 2$. We then have $\Delta O_{1}$ and $\Delta_{s} U_{1}$ nonnegative since each $\Delta_{s} B_{n} \geq 0$ by (16) and each $\Delta_{s} B_{n}^{\prime} \geq 0$ by (15). In addition, $\Delta_{s} B^{\prime} \geq 0$ for any box $B^{\prime} \subset L_{1}$ with left edge in $Y$; so, in particular, $\Delta_{s} L_{1} \geq 0$. If $\Delta_{s} R_{1} \geq 0$ we are done by (11).

Otherwise we iterate the process described in the preceding paragraph to obtain a nested sequence of boxes $\left\{R_{n}\right\}$, each $R_{n}$ having right edge contained in the right edge of $N(\gamma+t)$ as follows: having constructed $R_{n-1}$, perform the process inside the box $L_{n-1} \cup R_{n-1}$ with $V_{1}$ chosen to be the left half of $R_{n-1}$. If $\Delta_{s} R_{n} \geq 0$, we are done by (11). If for each $n=1,2, \ldots, \Delta_{s} R_{n}<0$, then $\bigcap R_{n}$ is a line segment (possibly degenerate) contained in the right edge of $N(\gamma+t)$, so that $\Delta_{s} N(\gamma+t) \geq 0$ by the continuity of $f$. 0 .

Applying the same argument to the left half of $B$ and using (11) gives $\Delta_{S} B \geq$

Case 2: $Y \cap B^{\circ} \cap C=\varnothing$. For $0<\varepsilon<b$, let $B_{\varepsilon}$ be the box centered at $(0,0)$ with first quadrant vertex $(a, b-\varepsilon)$. We apply Case 1 to get $\Delta_{s} B_{\varepsilon} \geq 0$. Let $\varepsilon \rightarrow 0$ and, by the continuity of $f$, we get $\Delta_{s} B \geq 0$. 
Case 3: $Y \cap B^{\circ} \cap C \neq \varnothing$. Let $V_{1}=B$ and pick $\left(x_{1}, y_{1}\right) \in Y \cap V_{1}^{\circ} \cap C$ closest to the horizontal bisector of $V_{1}$. Let $B_{1}$ be the largest box centered at $\left(x_{1}, y_{1}\right)$ contained in $V_{1}$. Suppose $V_{k},\left(x_{k}, y_{k}\right)$, and $B_{k}, k=1, \ldots, n-1$, have been selected. We let $V_{n}=\overline{V_{n-1} \backslash B_{n-1}}$ and pick $\left(x_{n}, y_{n}\right) \in Y \cap V_{n}^{\circ} \cap C$ closest to the horizontal bisector of $V_{n}$. Let $B_{n}$ be the largest box centered at $\left(x_{n}, y_{n}\right)$ and contained in $V_{n}$. This generates the sequence $\left\{B_{n}\right\}$, which is finite if some $Y \cap V_{n}^{\circ} \cap C=\varnothing$. If $\overline{\cup B_{n}}=B$, then $\Delta_{s} B \geq 0$ by the continuity of $f$. Otherwise, $B^{\prime}=\overline{B \backslash \bigcup B_{n}}$ is a box to which we apply Case 1 or 2 . Again, the continuity of $f$, along with the fact that $\Delta_{s} B^{\prime} \geq 0$, gives $\Delta_{s} B \geq 0$.

\section{TRIGONOMETRIC SERIES (MOTIVATION)}

The cornerstone to the theory of uniqueness of trigonometric series is the following theorem.

Theorem C (Cantor, 1870). If $\lim _{N \rightarrow \infty} \sum_{m=-N}^{N} c_{m} e^{i m x}=0$ for every real $x$, then every $c_{m}=0$.

Many extensions of this theorem have been found and even more have been proposed. (See, for some examples, the survey paper of Ash [1].) We wish to focus here on two particular extensions which are in the (long standing) conjectural condition.

Consider again the multiple trigonometric series $S(\mathbf{x})=\sum c_{\mathbf{n}} e^{i \mathbf{n} \mathbf{x}}$ and say that $S(\mathbf{x})$ converges spherically at $\mathbf{x}$ to $s$ and write $(\mathbf{S}) \sum c_{\mathbf{n}} e^{i \mathbf{n x}}=s$ if

$$
\lim _{M \rightarrow \infty} \sum_{|\mathbf{n}| \leq M} c_{\mathbf{n}} e^{i \mathbf{n} \mathbf{x}}=s .
$$

(Compare the definition of unrestricted rectangular convergence given in the introduction.)

Conjecture SC. If (S) $\sum c_{\mathbf{n}} e^{i \mathbf{n x}}=0$ for every $\mathbf{x}$ in $\mathbf{R}^{d}$, then every $c_{\mathbf{n}}=0$.

Conjecture AW. If $(\mathrm{R}) \sum c_{\mathbf{n}} e^{i \mathbf{n} \mathbf{x}}=0$ for every $\mathbf{x}$ in $\mathbf{R}^{d}$, then every $c_{\mathbf{n}}=0 .{ }^{1}$

If $d=1$ both these conjectures reduce to Theorem $\mathrm{C}$ and are therefore true. If $d=2$, both corijectures are again known to be true:

Theorem SC (Shapiro [13] and Cooke [7]). If (S) $\sum c_{\mathbf{n}} e^{i \mathbf{n x}}=0$ for every $\mathbf{x} \in \mathbf{R}^{2}$, then every $c_{\mathbf{n}}=0$.

Theorem AW (Ash and Welland [4]). If (R) $\sum c_{\mathbf{n}} e^{i \mathbf{n} \mathbf{x}}=0$ for every $\mathbf{x} \in \mathbf{R}^{2}$, then every $c_{\mathbf{n}}=0$.

For higher values of $d$, essentially nothing is known. Our main motivation for writing this paper has been our interest in Conjecture AW. A strange fact concerning the only currently known proof of Theorem AW is that the main lemma of the proof is (a generalization of) Theorem SC. We would very much like to find a direct rectangular proof of Theorem AW.

${ }^{1}$ Added in proof on December 15, 1992: This conjecture is proved in Uniqueness of rectangularly convergent trigonometric series, Ann. of Math. 137 (1993), 145-166, by J. Marshall Ash, Chris Freiling and Dan Rinne. 
We begin our study of Theorem AW by returning to dimension one. One proof of Theorem $\mathrm{C}$ begins by taking $S(x):=\sum c_{n} e^{i n x}$ and forming its formal integral $L(x):=c_{0} x+\sum^{\prime}\left(c_{n} /\right.$ in $) e^{i n x}$. (Here and later the prime means that all undefined terms, i.e., all terms with 0 denominator, are omitted from the summation.) [2] Although it is not clear from the hypothesis that the series for $L$ converges at every $x$, it can be shown that

(1) the series converges at almost every $x$,

(2) the limit function, call it $L(x)$ again, is an $L^{2}$ function, and

(3) $L(x)$ has an everywhere 0 symmetric first derivative in the $L^{2}$ sense.

The symmetric $L^{2}$ derivative is a special case of the symmetric approximate derivative and a recent differentiation theorem asserts that a measurable function with everywhere 0 symmetric approximate derivative is essentially constant (i.e., there is a constant to which the function is equal a.e. $[8,9])$. Once $L(x)$ is established to be essentially constant, it is very easy to get all the $c_{n}=0$. (This proof is the content of [2].) In summary, the above proof has three major steps:

I. Choose a formal integral to study.

II. A. Show that some generalized derivative of the formal integral is identically 0 .

B. Show that the formal integral is "good."

III. Prove a differentiation theorem that "good" functions with identically 0 generalized derivative have a special form.

Next we try to apply this three step scheme to achieve a new proof of Theorem AW. Assume that

$$
\text { (R) } \sum c_{\mathbf{n}} e^{i \mathbf{n} \mathbf{x}}=0 \text { for every } \mathbf{x} \text { in } \mathbf{R}^{2} .
$$

Beginning with step I we choose to study the formal integral.

By $\lim \operatorname{ap}_{(h, k) \rightarrow 0} g(h, k)=s$ we mean that there is a set $E \subset \mathbf{R}^{2}$ so that $\mathbf{0}=(0,0)$ is a point of density (with respect to two-dimensional Lebesgue measure) of $E$ and that $g(h, k)$ is close to $s$ provided (1) $\max \{|h|,|k|\}$ is small, (2) neither $h$ nor $k$ is 0 , and (3) $(h, k) \in E$.

Say that $L(x, y)$ has a symmetric approximate mixed partial derivative at $(x, y)$ equal to $s$ and write $D_{\text {sap }} f(x, y)=s$ if

$$
\lim \operatorname{ap}_{(h, k) \rightarrow 0}\left(\Delta_{s} f(x, y ; h, k) /(4 h k)\right)=s .
$$

It is an easy consequence of Tchebyshev's inequality that $D_{\text {sap }} L(x, y)$ exists and $D_{\text {sap }} f(x, y)=D_{s, 2} L(x, y)$ at each point where the latter derivative exists. Thus Theorem 3 (which will be proved below) achieves step II.A. in the program listed above. Now if a very good, $C^{2}$, say, function $L(\mathbf{x})$ satisfies $\left(\partial^{2} L / \partial x \partial y\right)(\mathbf{x})=0$ at every point $\mathbf{x}=(x, y)$, then it is necessarily of the form $a(x)+b(y)$. If we knew that the function $L$ of Theorem 3 had this $a(x)+b(y)$ form it would be very easy to finish the direct proof of Theorem AW.

In short, we need to find a definition of "good" which on the one hand is weak enough so that from (17) we can infer that $L$ is good, and on the other hand is strong enough so that all good $L(x, y)$ satisfying $D_{s, 2} L \equiv 0$ are necessarily of the form $a(x)+b(y)$.

Now from (17) the Cantor-Lebesgue Theorem [4, p. 408] implies that the coefficient of $L$ are $o\left(\frac{1}{m n}\right)$. In the one-dimensional situation, the function 
analogous to $L$ has coefficients $o\left(\frac{1}{n}\right)$. Just how good the trigonometric series is whose coefficients are $o\left(\frac{1}{n}\right)$ is the content of Theorem 4.

Proof of Theorem 3. We may suppose that $s=0$. Applying $\Delta_{s}$ term by term to $L$ yields

$$
\begin{aligned}
\frac{\Delta_{s} L(\mathbf{x} ; h, k)}{4 h k}= & c_{00}+\sum^{\prime} c_{m o} e^{i m x}\left(\frac{\sin m h}{m h}\right)+\sum^{\prime} c_{o n} e^{i n y}\left(\frac{\sin n k}{n k}\right) \\
& +\sum^{\prime} c_{m n} e^{i(m x+n y)}\left(\frac{\sin m h}{m h}\right)\left(\frac{\sin n k}{n k}\right) .
\end{aligned}
$$

The point of the hypothesis that the coefficient be bounded is to justify the calculation leading to equation (18). In fact, if $d_{m n}$ are the Fourier coefficients appearing in (18), then

$$
\begin{aligned}
& \sum\left|d_{m n}\right|^{2} \log (|m|+2) \log (|n|+2) \\
& \quad+C \sum \frac{\log (|m|+2 \mid) \log (|n|+2)}{(|m|+1)^{2}(|n|+1)^{2}}<\infty,
\end{aligned}
$$

so that, by a two-dimensional generalization [14] of a theorem of Kolmogorov and Seliverstov, Plessner, and Hardy and Littlewood (see [15, vol. II, XIII, 1.8, p. 162]), for almost every choice of $(h, k), L(x+h, y+k)$ exists and is the unrestricted rectangular sum of its Fourier series. It follows that formula (18) holds for almost every $(h, k)$. (Remark. This is the only use we make of the coefficient boundedness: that the right side of (18) converges in $L^{2}$ to $s=0$ will be shown below without reference to the magnitude of the coefficients. Notice that weaker conditions, such as $\left|c_{m n}\right| \leq|m n|^{0.4999}$, would suffice here.)

Write $A_{00}:=c_{00}, A_{m 0}:=c_{m 0} e^{i m x}+c_{-m 0} e^{-i m x}$ for $m>0, A_{0 n}:=c_{0 n} e^{i n y}+$ $c_{0-n} e^{-i n y}$ for $n>0$,

$$
\begin{aligned}
A_{m n}= & A_{m n}(\mathbf{x})=c_{m n} e^{i(m x+n y)} \\
& +c_{-m n} e^{i(-m x+n y)}+c_{m-n} e^{i(m x-n y)}+c_{-m-n} e^{-i(m x+n y)}
\end{aligned}
$$

for both $m$ and $n>0$, and $s_{m n}:=\sum_{\mu, \nu=0}^{m, n} A_{\mu \nu}$. Our hypothesis is now that

$$
\text { (R) } \sum_{m, n=0}^{\infty} A_{m n}=0 \text { and }\left|s_{m n}\right| \leq M
$$

and our goal is to show that

$$
\lim _{H, K \rightarrow 0} \frac{1}{H K} \int_{0}^{H} \int_{0}^{K}\left|\sum_{m, n=1}^{\infty} A_{m n} \frac{\sin m h}{m h} \frac{\sin n k}{n k}\right|^{2} d h d k=0
$$

We may assume that $H$ and $K$ are (negative) powers of 2 and then decompose $\int_{0}^{2^{-I}} \int_{0}^{2^{-J}}=\sum_{i, j=I, J}^{\infty} \int_{2^{-i-1}}^{2^{-i}} \int_{2^{-j-1}}^{2^{-\jmath}}$. This shows that our theorem is proved if we can show

$$
2^{I+J} \int_{2^{-I-1}}^{-I} \int_{2^{-J-1}}^{2^{-J}}\left|\sum A_{m n} \sigma(m h) \sigma(n k)\right|^{2} d h d k=o(1),
$$


where $\sigma(x):=\sin x / x$ for $x \neq 0$ and $\sigma(0):=1$. Sum by parts to get

$$
\begin{aligned}
\sum A_{m n} \sigma(m h) \sigma(n k)= & \lim _{M, N \rightarrow \infty} \sum_{\mu, \nu=0}^{M, N} s_{\mu \nu} \Delta \sigma(\mu h) \Delta \sigma(\nu k) \\
& +\lim _{M, N \rightarrow \infty} \sum_{\mu=0}^{M} s_{\mu N} \Delta \sigma(\mu h) \sigma(N k) \\
& +\lim _{M, N \rightarrow \infty} \sum_{\nu=0}^{N} s_{\mu \nu} \sigma(M h) \Delta \sigma(\nu k) \\
& +\lim _{M, N \rightarrow \infty} s_{M N} \sigma(M h) \sigma(N k) \\
:= & \mathrm{I}+\mathrm{II}+\mathrm{III}+\mathrm{IV},
\end{aligned}
$$

where $\Delta \sigma(\nu h):=\sigma(\nu h)-\sigma((\nu+1) h)$, etc. Clearly IV $=0$ and II and III are symmetric so it suffices to treat I and II.

We will do $I$ in detail and leave the easier II to the reader. As soon as $M$ and $N$ are large enough we may write

$$
\sum_{\mu, \nu=0}^{M, N}=\sum_{\mu, \nu=0}^{2^{I}, 2^{J}}+\sum_{\mu, \nu=0,2^{J}+1}^{2^{I}, N}+\sum_{\mu, \nu=2^{I}+1,0}^{M, 2^{J}}+\sum_{\mu, \nu=2^{I}+1,2^{J}+1}^{M, N}=: A+B+C+D .
$$

By symmetry, we need study $A, B$, and $D$ only. Since $|\Delta \sigma(\mu h)| \leq R|h|$, where $R:=\sup \left|\frac{d}{d x} \sigma(x)\right|$, we have by virtue of hypothesis (19)

$$
\begin{aligned}
|A| & =\left|\sum_{\mu, \nu=0}^{2^{I}, 2^{J}} s_{\mu \nu} \Delta \sigma(\mu h) \Delta \sigma(\nu k)\right| \\
& \leq R^{2}|h||k| \sum_{\mu, \nu=0}^{2^{l}, 2^{J}}\left|s_{\mu \nu}\right| \leq R^{2} \frac{1}{2^{I+J}} \sum_{\mu, \nu=0}^{2^{I}, 2^{J}}\left|s_{\mu \nu}\right|=o(1) .
\end{aligned}
$$

To study $D$, write

$$
\begin{aligned}
\Delta \sigma(\mu h) & =\left\{\sigma(\mu h)-\frac{\sin \mu h}{(\mu+1) h}\right\}+\left\{\frac{\sin \mu h}{(\mu+1) h}-\sigma((\mu+1) h)\right\} \\
& =\frac{\sin \mu h}{\mu(\mu+1) h}-\frac{2 \sin \frac{h}{2} \cos \left(\mu+\frac{1}{2}\right) h}{(\mu+1) h}
\end{aligned}
$$

and similarly expand $\Delta \sigma(\nu k)$ to get

$$
\begin{aligned}
D= & \sum s_{\mu \nu} \frac{\sin \mu h}{\mu(\mu+1) h} \frac{\sin \nu k}{\nu(\nu+1) k}-\sum s_{\mu \nu} \frac{\sin \mu h}{\mu(\mu+1) h} \frac{2 \sin \frac{k}{2}}{k} \frac{\cos \left(\nu+\frac{1}{2}\right) k}{(\nu+1)} \\
& -\sum s_{\mu \nu} \frac{2 \sin \frac{h}{2}}{h} \frac{\cos \left(\mu+\frac{1}{2}\right) h}{\mu+1} \frac{\sin \nu k}{\nu(\nu+1) k} \\
& +\sum s_{\mu \nu} \frac{2 \sin \frac{h}{2}}{h} \frac{2 \sin \frac{k}{2}}{k} \frac{\cos \left(\mu+\frac{1}{2}\right) h}{\mu+1} \frac{\cos \left(\nu+\frac{1}{2}\right) k}{\nu+1} \\
=: & D_{1}-D_{2}-D_{3}+D_{4} .
\end{aligned}
$$


We have

$$
\begin{aligned}
\left|D_{1}\right| & =\left|\sum_{\mu, \nu=2^{I}+1,2^{J}+1}^{M, N} s_{\mu \nu}\left(\frac{\sin \mu h}{\mu h}\right)\left(\frac{\sin \nu k}{\nu k}\right) \frac{1}{(\mu+1)(\nu+1)}\right| \\
& \leq \frac{1}{|h k|} \sum \frac{\left|s_{\mu \nu}\right|}{\mu(\mu+1) \nu(\nu+1)} \leq 4 \cdot 2^{I+J} \sum \frac{\left|s_{\mu \nu}\right|}{\mu(\mu+1) \nu(\nu+1)}=o(1)
\end{aligned}
$$

since $\left|s_{\mu \nu}\right|$ is small on the average and bounded.

To this point we have been making routine estimates and the $L^{2}$ theory has not been relevant. On the next term we use Parseval's formula:

$$
\begin{aligned}
\int_{2^{-J}-1}^{2^{-J}} & \left|D_{2}\right|^{2} d k \\
& \leq \int_{0}^{\pi}\left|\sum_{\nu=2^{j}+1}^{N}\left\{\frac{1}{\nu+1}\left(\sum_{\mu=2^{I}+1}^{M} s_{\mu \nu} \frac{\sin \mu h}{\mu(\mu+1) h}\right)\right\} \cos \left(\nu+\frac{1}{2}\right) k\right|^{2} d k \\
& =\frac{\pi}{2} \sum_{\nu=2^{J}+1}^{N}\left|\frac{1}{\nu+1}\left(\sum_{\mu=2^{I}+1}^{M} s_{\mu \nu} \frac{\sin \mu h}{\mu(\mu+1) h}\right)\right|^{2} .
\end{aligned}
$$

But

$$
\sum_{\mu=2^{I}+1}^{\infty} \frac{\left|s_{\mu \nu}\right|}{\mu(\mu+1)}=o(1) \cdot \frac{1}{2^{I}}
$$

and $1 /|h|=O\left(2^{I}\right)$ so that $\int_{2^{-J-1}}^{2^{-J}}\left|D_{2}\right|^{2}$ is $o\left(\sum_{\nu=2^{J}+1}^{\infty} 1 /(\nu+1)^{2}\right)=o\left(1 / 2^{J}\right)$. Average in $h$ over $\left[2^{-I-1}, 2^{-I}\right]$ to achieve $(20)$ for the portion of the integral assigned to $D_{2}$. The estimate for $D_{3}$ is symmetrical to that for $D_{2}$. Now the estimate for $D_{4}$ uses the Parseval formula in two variables:

$$
\begin{aligned}
& \int_{2^{-I-1}}^{2^{-I}} \int_{2^{-J-1}}^{2^{-J}}\left|D_{4}\right|^{2} d h d k \\
& \leq \sup \left|\frac{2 \sin \frac{h}{2}}{h} \frac{2 \sin \frac{k}{2}}{k}\right|^{2} \int_{0}^{\pi} \int_{0}^{\pi} \mid \sum_{\mu, \nu=2^{I}+1,2^{J}+1}^{M, N} \frac{s_{\mu \nu}}{(\mu+1)(\nu+1)} \\
& \left.\quad \cdot \cos \left(\mu+\frac{1}{2}\right) h \cos \left(\nu+\frac{1}{2}\right) k\right|^{2} d h d k \\
& \leq\left(\frac{\pi}{2}\right)^{2} \sum_{\mu, \nu=2^{I}+1,2^{J}+1}^{\infty} \frac{\left|s_{\mu \nu}\right|^{2}}{(\mu+1)^{2}(\nu+1)^{2}}=o(1) \cdot 2^{-I-J} .
\end{aligned}
$$

Thus the contribution of $D$ to the left side of (20) is controlled.

Only the term $B$ remains. Here we split into two terms by writing

$$
\Delta \sigma(\nu k)=\frac{\sin \nu k}{\nu(\nu+1)}-\frac{2 \sin \frac{k}{2}}{k} \frac{\cos \left(\nu+\frac{1}{2}\right) k}{\nu+1}
$$

and show that the terms containing $\sin \nu k / \nu(\nu+1)$ go to 0 directly while the other terms are treated by applying Parseval's formula in the $k$ variable. 
Remarks. This proof of Theorem 3 follows the proof of a one-dimensional version of Theorem 3, due to Rajchman and Zygmund [15, vol. I, p. 324]. If $\mathbf{x} \in \mathbf{R}^{d},(\mathbf{R}) \sum c_{\mathbf{n}} e^{i \mathbf{n x}}=s,\left|s_{\mathbf{n}}(\mathbf{x})\right| \leq M$, and $c_{\mathbf{n}}$ are bounded, then $L(\mathbf{x})$, the function obtained by formally integrating once in each variable, has a generalized $\partial^{d} / \partial x_{1} \partial x_{2} \cdots \partial x_{d}$ symmetric approximate derivatives at $\mathbf{x}$ equal to $s$. This is clear since it is easy to see that the above proof was not at all constrained by dimension. All that is needed is more notation.

Proof of Theorem 4. Let $b(x)=\sum b_{n} e^{i n x}$, where $n b_{n} \rightarrow 0$ as $|n| \rightarrow \infty$. Let $T_{y}$ be translation by $y$. It suffices to show that

$$
\left\|T_{y} b-b\right\|_{*}:=\sup _{\|h\|_{H^{1}} \leq 1}\left(h, T_{y} b-b\right) \rightarrow 0
$$

as $y \rightarrow 0\left[12\right.$, p. 392]. Fix an $h$ in the unit ball of $H^{1}$ and let $\varepsilon>0$ be given. Pick $N$ so large that $\sup _{|n| \geq N}\left|n \bar{b}_{n}\right| \leq \varepsilon / 2$. Then pick $y$ so small that $\left|1-e^{-i k y}\right| \leq \varepsilon / M$ for every $k$ with $|k|<N$, where $M=\sup _{n}\left|n \bar{b}_{n}\right|$. Then

$$
\begin{aligned}
\left(h, T_{y} b-b\right) & =\sum \frac{h_{n}}{n}\left(n b_{n}\right)\left(1-e^{-i n y}\right) \\
& \leq \sum_{|n|<N}\left|\frac{h_{n}}{n}\right| \cdot M \cdot \frac{\varepsilon}{M}+\sum_{n \geq N}\left|\frac{h_{n}}{n}\right| \cdot \frac{\varepsilon}{2} \cdot 2 \\
& \leq \varepsilon \sum\left|\frac{h_{n}}{n}\right| \leq \varepsilon\|h\|_{H^{1}} \leq \varepsilon .
\end{aligned}
$$

The penultimate inequality here can be found in [15, vol. I, p. 286]. Hence $b \in \mathrm{VMO}$.

Remark. To get some sense of the sharpness of Theorem 2, consider the saw tooth function which is the $2 \pi$ periodic extension of the function defined as $(\pi \operatorname{sgn} x-x) / 2$ on $[-\pi, \pi)$. This function has Fourier series $\sum \sin n x / n$. The Fourier coefficients just barely fail to be $o\left(\frac{1}{n}\right)$ and the function (just barely) fails to be everywhere $\left(L^{p}, 1 \leq p \leq \infty\right.$, say) continuous due to the jump at $x=0$. This function provided the motivation for Theorem 2. E. Stein told us that we could improve our original conclusion of $L^{2}$ symmetric continuity to membership in VMO.

Remark. In particular, a function whose Fourier coefficients are $o\left(\frac{1}{n}\right)$ is symmetrically continuous in the $L^{p}$ sense, $1 \leq p<\infty$, and in the approximate sense, at every point.

Proof. Fix any point $x$, let $I:=[x-h, x+h]$ and $b_{I}=\frac{1}{2 h} \int_{I} b$, and apply the triangle inequality to get

$$
\begin{aligned}
& \left(\frac{1}{2 h} \int_{-h}^{h}|b(x+t)-b(x-t)|^{p} d t\right)^{1 / p} \\
& \quad \leq\left(\frac{1}{2 h} \int_{-h}^{h}\left|b(x+t)-b_{I}\right|^{p} d t\right)^{1 / p}+\left(\frac{1}{2 h} \int_{-h}^{h}\left|b_{I}-b(x-t)\right|^{p} d t\right)^{1 / p} .
\end{aligned}
$$

By the definition VMO, both of the integrals on the right tend to 0 as $h$ tends to 0 , so that $b$ is symmetrically $L^{p}$ continuous at $x$. This always implies 
symmetric approximate continuity because of Tchebychef's inequality. For an alternative proof of the fact that having $o\left(\frac{1}{n}\right)$ Fourier coefficients forces a function to be symmetrically approximately continuous see Rajchman and Zygmund [11].

\section{FutURE DIRECTIONS}

In view of Theorems 2, 3, and 4, there seems to be real hope for at least an honestly rectangular proof of uniqueness in two dimensions. For example, the following pair of conjectures coupled with Theorem 3 would do the job.

Conjecture 1. If $(\mathrm{R}) \sum c_{\mathbf{n}} e^{i \mathbf{n x}}=0$ for all $\mathbf{x}$ in $\mathbf{R}^{2}$, then $L(\mathbf{x})$ is of vanishing mean oscillation.

Conjecture 2. If $D_{\text {sap }} L(\mathbf{x})=0$ for all $\mathbf{x}=(x, y)$ in $\mathbf{R}^{2}$ and if $L(\mathbf{x})$ is of vanishing mean oscillation, then $L(\mathbf{x})$ is essentially of the form $a(x)+b(y)$.

The one-dimensional proof of uniqueness in [2] relied on a generalization $[8,9]$ of the property that for smooth $f, f^{\prime}=0$ implies that $f$ is constant. As mentioned in the previous paragraph, the next goal is a sufficiently broad generalization of the fact that for smooth $f, \partial^{2} f / \partial x \partial y=0$ implies that $f(x, y)=a(x)+b(y)$. Ultimately we will need a sufficiently broad generalization of the fact that for smooth $f, \partial^{d} f / \partial x_{1} \partial x_{2} \cdots \partial x_{d}=0$ implies $f(x)=f_{1}\left(x_{2}, \ldots, x_{d}\right)+f_{2}\left(x_{1}, x_{3}, \ldots, x_{d}\right)+\cdots+f_{d}\left(x_{1}, \ldots, x_{d-1}\right)$. Such a result would provide a reductive proof of Conjecture AW of $\S 5$.

\section{ACKNOWLEDGMENTS}

The authors wish to thank the referee for his careful reading and many helpful suggestions. The authors are grateful to Erich A. Krausser for his help with the plotting of Figure 2.

\section{REFERENCES}

1. J. M. Ash, Uniqueness of representation by trigonometric series, Amer. Math. Monthly 96 (1989), 873-885.

2. __ A new proof of uniqueness for multiple trigonometric series, Proc. Amer. Math. Soc. 107 (1989), 409-410.

3. J. M. Ash, A. E. Gatto, and S. Vàgi, A multidimensional Taylor's theorem with minimal hypothesis, Colloq. Math. 60-61 (1990), 245-252.

4. J. M. Ash and G. V. Welland, Convergence, uniqueness, and summability of multiple trigonometric series, Trans. Amer. Math. Soc. 163 (1972), 401-436.

5. K. Bogel, Über die mehrdimensionale Differentiation, Jber. Deutsch. Math.-Verein. 65 (1962), 45-71.

6. Z. Buczolich, A general Riemann complete integral in the plane, Acta Math. Hungar. 57 (1991), 315-323.

7. R. Cooke, A Cantor-Lebesgue theorem in two dimensions, Proc. Amer. Math. Soc. 30 (1971), 547-550.

8. C. Freiling and D. Rinne, A symmetric density property, monotonicity and the approximate symmetric derivative, Proc. Amer. Math. Soc. 104 (1988), 1098-1102.

9. __ A symmetric density property for measurable sets, Real Anal. Exchange 14 (1988-89), 203-209. 
10. A. Khintchine, Recherches sur la structure des fonctions mesurables, Fund. Math. 9 (1927), 212-279.

11. A Rajchman and A. Zygmund, Sur la relation du procédé de sommation de Cesàro et celui de Riemann, Bull. Internat. Acad. Polon. Sér. A (1925), 69-80. (Also in Selected Papers of Antoni Sygmund, Vol. 3 (A. Hulanicki et al., eds., Kluwer, Dordrecht, 1989, pp. 81-92.)

12. D. Sarason, Functions of vanishing mean oscillation, Trans. Amer. Math. Soc. 207 (1975), 391-405.

13. V. L. Shapiro, Uniqueness of multiple trigonometric series, Ann. of Math. (2) 66 (1957), 467-480.

14. L. V. Zhizhiashvili, Some problems in the theory of simple and multiple trigonometric series, Russian Math. Surveys 28 (1975), 65-127.

15. A. Zygmund, Trigonometric series, Vols. 1, 2, 2nd rev. ed., Cambridge Univ. Press, New York, 1959.

(J. M. Ash and J. Cohen) Department of Mathematics, DePaul University, Chicago, ILLINOIS 60614

E-mail address: matjma@depaul.bitnet

E-mail address: matjc@depaul.bitnet

(C. Freiling and D. Rinne) Department of Mathematics, California State University, San Bernardino, California 92407

E-mail address, D. Rinne: drinne@wiley.csusb.edu 